\title{
The epidemiology series of brain-related disorders in Mongolia: nationwide registry-based epidemiological study on CNS tumours in Mongolia, 2015-2019
}

\author{
Tuvshingerel Sandagdorj ${ }^{1}$, Undarmaa Tudev ${ }^{2}$, Otgonbold Jamiyandorj ${ }^{3}$, Takakazu Oka ${ }^{4}$, Battuvshin Lkhagvasuren ${ }^{4,5, *}$ \\ ${ }^{1}$ Division for Planning, Monitoring, and Economics, National Cancer Center, 13370 Ulaanbaatar, Mongolia \\ ${ }^{2}$ Division for Cancer Registry and Screening, National Cancer Center, 13370 Ulaanbaatar, Mongolia \\ ${ }^{3}$ School of Dentistry, Mongolian National University of Medical Sciences, 14210 Ulaanbaatar, Mongolia \\ ${ }^{4}$ Department of Psychosomatic Medicine, International University of Health and Welfare Narita Hospital, 286-8520 Chiba, Japan \\ ${ }^{5}$ Brain Science Institute, Graduate School, Mongolian National University of Medical Sciences, 14210 Ulaanbaatar, Mongolia \\ *Correspondence: battuvshin@mnums.edu.mn (Battuvshin Lkhagvasuren)

\section{DOI:10.31083/j.jin2101024} \\ This is an open access article under the CC BY 4.0 license (https://creativecommons.org/licenses/by/4.0/). \\ Submitted: 1 May 2021 Revised: 28 July 2021 Accepted: 6 August 2021 Published: 28 January 2022
}

No epidemiological study on central nervous system tumours is available for Mongolia. The aim of this study was to determine the incidence, mortality and survival of people diagnosed with central nervous system tumours in Mongolia. It reports cancer data for the entire population (3.3 million) during the period between 2015 and 2019. Data was obtained from the National Cancer Registry of Mongolia. Diagnosis of tumours was established according to the diagnostic criteria of the International Classification of Diseases-10 (ICD10). Incidence and mortality rates were calculated as mean annual numbers per 100,000 population. Age-standardized incidence and age-standardized mortality rates were calculated from age-specific rates by weighting directly from the World Standard Population. The three-year survival from 2015 through 2017 was calculated between treatment types by the Kaplan-Meier survival analysis. It found 515 (adults: $83 \%$; children: 17\%) newly diagnosed central nervous system tumour cases over the five year period. The national agestandardized incidence of central nervous system tumours for the entire population was 3.7 per 100,000 . The rate was higher for males than females ( 4.2 versus 3.4 per 100,000 , respectively). Only $23 \%$ of the diagnosed cases were confirmed histologically. The most common tumour was glioma ( $57.6 \%$ of histologically verified tumours). In children (age 0--19 years) the age-specified incidence rate of tumours was 1.4 per 100,000. Ceographically, the age-standardized incidences of the Eastern region were higher than the country average rates for both genders. During the period, 381 deaths were registered with an age-standardized mortality rate of 3.0 per 100,000 population. Furthermore, the overall three-year survival rate was $40.6 \%$ (out of 283 patients, 115 survived). The five-year prevalence of tumours was 183 and the mean per 100,000 population was 5.5. In conclusion, the data from the National Cancer Registry indicate that the incidence and survival rates of central nervous system tumours in Mongolia are relatively low. The most common location of central nervous system tumours was the brain. Clioma was the most common tumour among histologically confirmed cases. Despite the limitations, data from this study should provide information for national health policy and health care assessment. To improve the diagnosis, prognosis and treatment of central nervous system tumours, expansion of the cancer registry through collecting data on non-malignant tumours, increasing the rate of histological verification, conducting studies on cancer epidemiology and the introduction of advanced treatment technologies for central nervous system tumours are recommended.

\section{Keywords}

Brain-related disorders; CNS tumours; Age-standardized incidence rate; Agestandardized mortality rate; Mongolia

\section{Introduction}

Mongolia is located between China and Russia, but distinct from either both ethnically and culturally. It has a small population (3.3 million) but a large geographic area. Mongolia is classified as a lower-middle income economy by the World Bank [1]. The country is divided into four regions: Western, Mountain (Khangai), Central and Eastern. Ulaanbaatar, the capital city with half of the total population, is counted separately. The Mongolian population is relatively young, with $38 \%$ of people aged $0-19$ years. More than half of this fairly homogenous population lives in rural areas in a Ger (a nomadic pelt tent) as nomadic herdsmen. Gers are not connected to water, electricity, sanitation, or heating systems. Recent migration of rural population into Ulaanbaatar has expanded its Ger areas where $60 \%$ of the residents live [2] in one of the most air-polluted cities in the world [3].

The health care system is divided into three levels of services. Primary health care is provided through 334 primary health centers distributed throughout Mongolia. Secondary health care is delivered through general hospitals: seven in the capital city, eighteen in the prefectures and three in the regions [4]. Any suspicious case of tumour detected at a primary health center is referred to a secondary health care hospital. Each secondary hospital provides outpatient services for cancer patients. For diagnosis verification, treat- 
ment and palliative care, patients are further referred to tertiary health care centers. Tertiary health care is provided through the National Centers for main medical disciplines. The National Cancer Center (NCC) serves the entire population with specialized health care in oncology by prevention, early detection, registry, diagnosis and treatment. It was established in 1961 and is the only hospital in the country that provides radiation therapy and chemotherapy for cancer. The department of radiotherapy includes eight oncologists, sixteen registered nurses, and ten radiology technicians and offers conventional radiotherapy with a 3D Cobalt type teletherapy unit (Bhabhatron 2, India), a Cobalt type Teletherapy (GWHJ-80, China), a Simulator X-ray (HMD1B, China), a Simulator X-ray (Imagin, India) and a Simulator CT scan (Somatom Emotion 6, Siemens, Germany). The department of radiology includes four radiologists, two registered nurses and four technicians and has a computer tomography (CT) scan unit (Somatom, Siemens, Germany) and four X-rays (Heliodent, Mobilett, Siemens, Germany). Additionally to the NCC, the Third General Hospital of Mongolia (TGHM) is the only health care center that provides surgical therapies for central nervous system (CNS) tumours in the country. The department of neurosurgery has nineteen neurosurgeons, twenty-five registered nurses and seven technicians. Therefore, patients with CNS tumours are mostly diagnosed in the secondary health care hospitals, treated in the tertiary health care centers and sent back for monitoring to a secondary health care hospital for specialized medical services. If patients seek medical help abroad after diagnosis verification, their diagnoses are registered but the outcome is not recorded at the National Cancer Registry of Mongolia (NCRM). Due to the limitations in diagnostic and management resources, patients are encouraged to visit other public and private hospitals for diagnostic purposes. Some newly established hospitals such as National Diagnostic Center and the Mongolia-Japan Teaching Hospital have magnetic resonance imaging systems ( $3 \mathrm{~T}$ Vantage, Canon, Japan, or equivalent), CTs (64-slice Aquilion, Toshiba or equivalent) and other diagnostic equipment.

NCRM is the nationwide cancer registry located at the NCC. NCRM collects data on newly-diagnosed malignant tumours, deaths due to malignant tumours and treatment care for malignant tumours from all hospitals through both passive and active case-finding methods. It does not collect data for non-malignant tumours. Active registration is supplementary to passive registration through occasional visits by registrars to pathology laboratories in tertiary care centers. Cases are detected through death certificates in cases where the death occurred at home. The records are sent to statistical units of geopolitical administration offices, then compiled and reported to the respective departments of the General Authority for State Registration and the Ministry of Health. NCRM uses a registry software program, CANREGMON, which is based on CanReg4 software produced by the International Agency for Research on Cancer. It is an open- source software package for cancer registries to enter, quality control and store data on cancer according to international guidelines. Duplications are eliminated using an unique citizen ID number. All hospitals must report new cancer cases and deaths from cancer to the NCRM following the Cancer Registry Guideline with a notification form (\#AM5) approved by the Ministry of Health. NCRM collects all data on malignant tumours and sends annual reports to the Health Development Center of Mongolia (HDCM). A major disadvantage of NCRM is its inability to register non-malignant tumours. There is no place to register benign tumours in Mongolia. Therefore, the term of CNS tumour employed in this article represents only malignant CNS tumours registered at NCRM. Another limitation is that the pathological laboratories in secondary and tertiary health care belong to the National Pathology Center (NPC) which does not report histological examination results of newly diagnosed tumours to the NCRM. The pathology laboratory of NCC does not examine CNS tumours. Generally, cancer diagnoses should be based on the results of imaging techniques, i.e., in most cases by CT and MRI in Mongolia, then verified by histological examination. However, in Mongolia, diagnoses of CNS tumours are mostly based on imaging technique results, since the pathology laboratory of the TGHM does not report their histological findings of the neurosurgery patients who underwent a surgical operation. Also, NCRM registers CNS tumour diagnosis based on imaging results only without histological confirmation if a patient dies shortly after a diagnosis. This is reasonable since postmortem diagnoses of cancer are very rare in Mongolia due to cultural and religious factors. Therefore, it is speculated that the histologically confirmed diagnosis rate of CNS tumours in Mongolia may be very low compared to other countries.

Globally, CNS tumours have increased by almost $20 \%$ in recent decades. East Asia, in particular, had the most incident cases of CNS tumours regardless of sex [5]. In the Asia-Pacific region, the countries with the highest age-standardized incidence rates of CNS tumours were China, India, Australia and New Zealand [6]. As Global Cancer Incidence, Mortality, and Prevalence (Globocan) 2018 reported, the worldwide incidence rate and mortality rate of malignant CNS tumours were 3.5 and 2.8 per 100,000 population, respectively [7].

According to the annual reports on health indicators developed by HDCM, cancer-related disease burden and mortality have dramatically increased in recent decades. Deaths from cancer account for $24 \%$ of male and $30 \%$ of female deaths [4]. Mongolians have the highest age-standardized cancer mortality rate in the Asia Pacific region [8] which is associated with late-stage diagnosis, low survival rate and limited coverage of health care $[8,9]$. For instance, according to a global cancer survival study, Mongolia had among the lowest five-year survival estimates for lung and liver cancers [9] and the incidence of liver cancer was one of the highest in the world [10-13]. Similarly, it is speculated that survival of CNS tumours might also be very low. 
These unique characteristics may raise particular interest in the epidemiology of CNS tumours in Mongolia. Despite a low incidence rate, CNS tumours represent a considerable burden of morbidity and mortality worldwide [13].

There has been no study on the epidemiological and clinical characteristics of CNS tumours in Mongolia. Therefore, this study provides quantitative data on the distribution of CNS tumours and can inform awareness of the differences between geographical regions and survival rates. Increased understanding of the epidemiology of CNS tumours might provide information for health policy and medical services toward better treatment, diagnosis and prevention. The purpose of this article is to describe the national incidence, mortality and survival of CNS tumours for 2015-2019 in Mongolia.

\section{Materials and methods}

\subsection{Data source}

The national cancer registry data from the NCRM was used. The study includes all newly diagnosed cases and deaths due to primary CNS tumours registered at NCRM from 2015 to 2019 for the entire population from birth to 95 years, divided into five-year age groups. In a period of five years a total of 515 patients were diagnosed with CNS tumours according to the International Classification of Diseases-10 (ICD10): Malignant neoplasm of brain (C71.0-C71.9); Malignant neoplasm of meninges (C70.0-C70.9); and Malignant neoplasm of spinal cord (C72.0-C72.9) [14]. Newly diagnosed cancer must be registered with a notification form to NCRM that includes either histological or radiological evidence. The childhood incidence rate was calculated separately for children aged between birth and nineteen. During the same period a total of 381 deaths attributed to CNS tumours were registered. Histological grouping was classified by the ICD-O-3 classification [15]. For a three-year survival analysis, the data from 283 patients diagnosed with CNS tumours from 2015 to 2017 were included. Data from 232 patients who were either diagnosed in 2018 and 2019 or were untraceable for follow up for three years were excluded. Population denominator data were drawn from the 2019 reports of the National Statistical Office of Mongolia [2].

\subsection{Statistical analysis}

Age-standardized incidence rates (ASRs) and agestandardized mortality rates (ASMRs) were calculated as the mean annual rates per 100,000 population. The direct age standardization method was employed to produce ASRs and ASMRs utilizing the World Standard Population [16]. Age-specific incidence rates were calculated according to eighteen age groups categorized by the national census with a five-year interval from birth. The ASRs were calculated by dividing the annual average of cases in a period between 2015 and 2019 by the average annual population in this period and were expressed per 100,000 population. Although the primary objective of this study was to determine the incidence rate, the five-year prevalence was also calculated
Table 1. Five-year new cases, mean annual age-standardized incidence rates by geographical region, 2015-2019 $(n=515)$.

\begin{tabular}{lcccccc}
\hline \multirow{2}{*}{ Characteristics } & \multicolumn{3}{c}{ All population } & \multicolumn{2}{c}{ Males } & \multicolumn{2}{c}{ Females } \\
\cline { 2 - 7 } & Cases & ASR & Cases & ASR & Cases & ASR \\
\hline Mongolia & 515 & 3.7 & 269 & 4.2 & 246 & 3.4 \\
Urban region & & & & & & \\
$\quad$ Ulaanbaatar & 213 & 3.5 & 105 & 3.7 & 108 & 3.3 \\
Rural region & & & & & & \\
$\quad$ Western & 55 & 3.1 & 25 & 2.6 & 30 & 3.4 \\
$\quad$ Mountain & 97 & 3.5 & 56 & 4.0 & 41 & 2.9 \\
$\quad$ Central & 87 & $4.0^{*}$ & 53 & $5.1^{*}$ & 34 & 3.0 \\
Eastern & 63 & $6.6^{*}$ & 30 & $7.6^{*}$ & 33 & $6.2^{*}$ \\
\hline
\end{tabular}

${ }^{*}$ Higher than the national rate $(3.7 / 100,000)$.

by considering the number of incident cases during the period between 2015 and 2019 who were alive at the end of 2019. The overall result for three-year survival was calculated between the type of treatment modalities (surgical treatment, radiotherapy/chemotherapy and treatment unknown) during the period of 2015-2017 by the log-rank test using the Kaplan-Meier method. Data are presented as mean \pm standard deviation (Mean \pm SD). Statistical significance was set at $p<0.05$ and all tests were two-tailed. Data were analyzed using SPSS v26.0 (IBM Corp., New York, NY, USA).

\section{Results}

3.1 Incidence rate

3.1.1 Age-standardized incidence rate

Between 2015 and 2019, a total of 515 CNS tumour cases (age range: $1-95$, children: $17 \%$, mean age: $46 \pm 23$, and median age: 50$)$ were reported to NCRM. $52 \%$ were male $(n=$ 269 , age range: $1-85$, mean age: $43 \pm 22$, median age: 47 ) and $48 \%$ were female ( $n=246$, age range: $1-95$, mean age: 49 \pm 23 , median age: 51 ).

By topographic distribution of CNS tumours, 436 (84.7\%) were diagnosed with malignant neoplasms of the brain, 60 (11.7\%) with malignant neoplasms of meninges and 19 (3.9\%) with malignant neoplasms of the spinal cord, respectively (Fig. 1). Among men, the leading site of CNS tumours was the brain (85.4\%). Among women, the rate of CNS tumours in the brain was slightly lower (83.8\%) than for men. In children, $90.8 \%$ of CNS tumours were malignant neoplasms of the brain.

Furthermore, by ICD-10 classification, the most common originating site in the brain was the cerebrum except lobes and ventricles (14\%), whereas $57 \%$ of newly diagnosed CNS tumours in the brain were not specified topographically (Fig. 1B).

The national ASR of all CNS tumours was 3.7 per 100,000 population (Table 1 ). This rate was higher in males compared to females (4.2 per 100,000 males; 3.4 per 100,000 females).

The ASRs in the Eastern region were higher than both the rates in other regions and the national rates. The Central region also had higher rates, whereas the Western and Moun- 


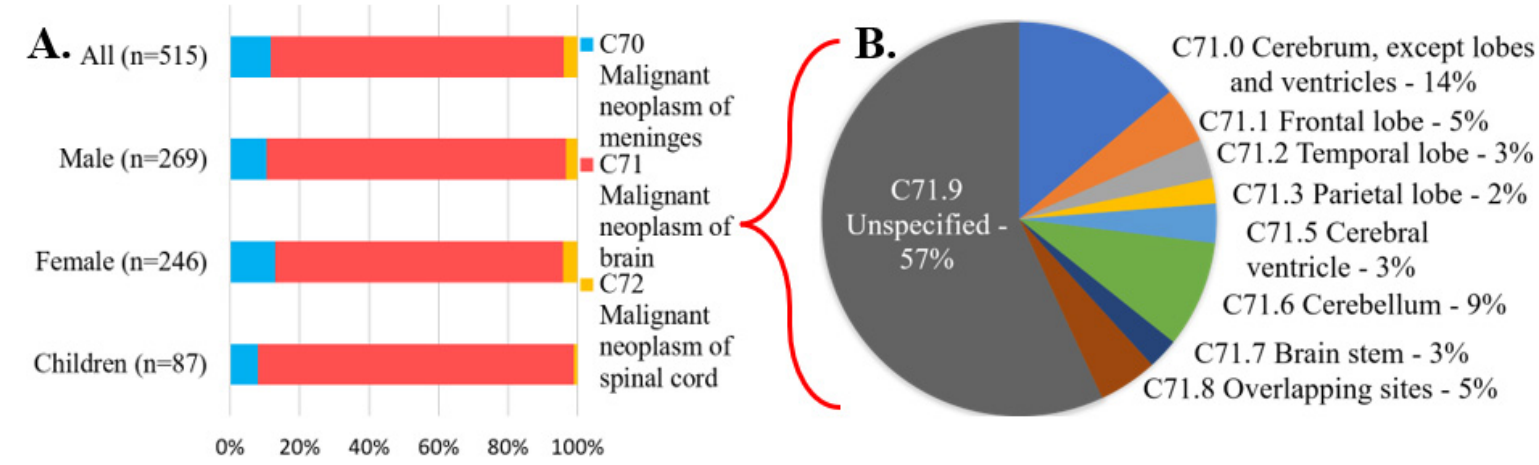

Fig. 1. Topographic distribution of CNS tumours. (A) Topographic distribution of all newly diagnosed CNS tumours according to the ICD-10 classification in adults by sex and in children. The malignant neoplasms of the brain were the most common site of CNS tumours. (B) Distribution of the malignant neoplasms of brain (C71) according to the ICD-10 classification by the originating site, 2015-2019. The cerebrum and cerebellum were the leading sites of the malignant neoplasms of the brain specified topographically.

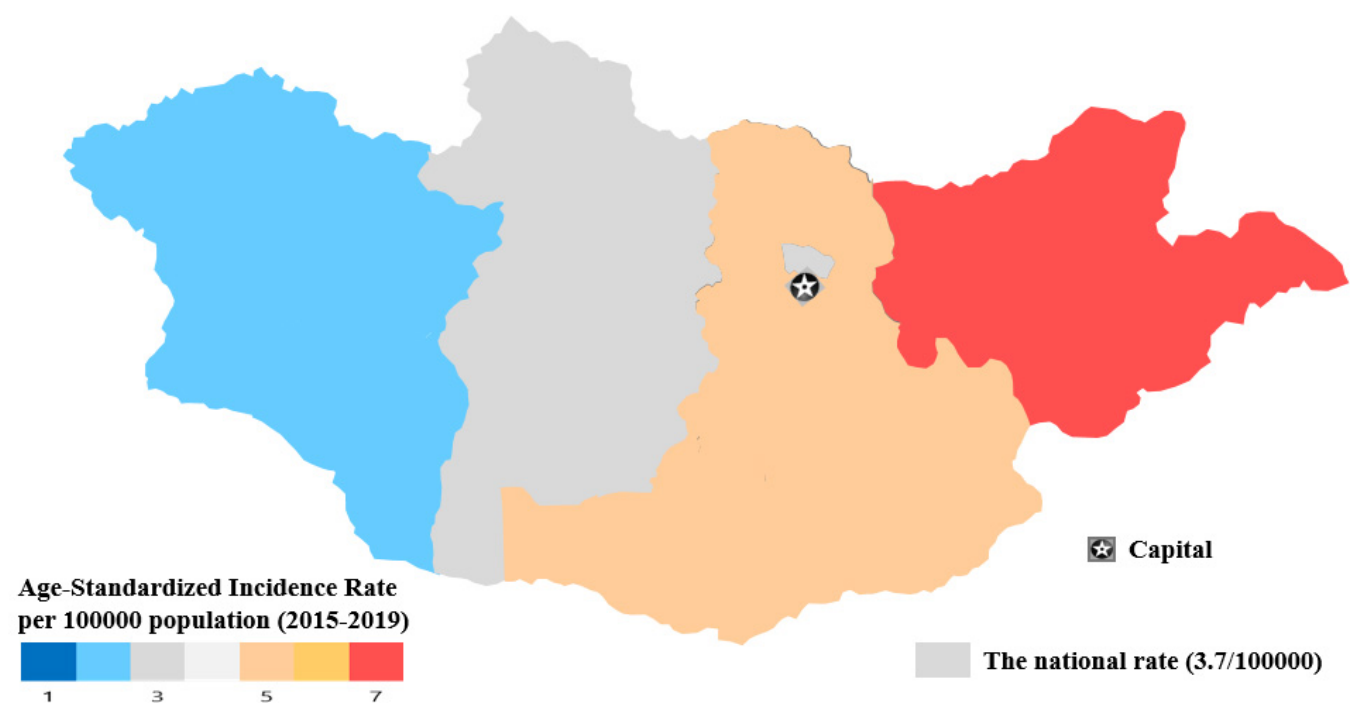

Fig. 2. The national mean annual age-standardized incidence rate of CNS tumours per 100,000 population, $2015-2019$.

tain regions had lower rates than the national rate (Fig. 2). Except for the Western region, the ASR was higher in males than females.

\subsubsection{Age-specific incidence rate}

The age-specific incidence rates were calculated according to eighteen age groups categorized by the national census (0-4, 5-9, 10-14, 15-19, 20-24, 25-29, 30-34, 35-39, 40$44,45-49,50-54,55-59,60-64,65-69,70-74,75-79,80-84$, $>85$ ).

Fig. 3 displays the age-specific curves of incidence rates for both sexes during 2015-2019 in Mongolia. During the period, most CNS tumours were diagnosed in the age group 65-69 years with the highest age-specific incidence rate in the ages 80-84 years for females and ages 55-59 for males.

\subsubsection{Childhood incidence rate}

A total of 87 cases (age range: $0-19$, mean age: $8.3 \pm 5.3$, median age: 7 ) of CNS tumours were diagnosed in children (Table 2).
Table 2. Five-year new cases and mean annual incidence rates per 100,000 children (0-19 years), 2015-2019 $(n=87)$.

\begin{tabular}{lcccccc}
\hline \multirow{2}{*}{ Characteristics } & \multicolumn{2}{c}{ All children } & \multicolumn{2}{c}{ Boys } & \multicolumn{2}{c}{ Girls } \\
\cline { 2 - 7 } & Cases & Rate & Cases & Rate & Cases & Rate \\
\hline Mongolia & 87 & 1.4 & 53 & 1.7 & 34 & 1.1 \\
Urban region & & & & & & \\
$\quad$ Ulaanbaatar & 33 & 1.2 & 20 & 1.4 & 13 & 1 \\
Rural region & & & & & & \\
$\quad$ Western & 9 & 1.1 & 5 & 1.2 & 4 & 1.0 \\
$\quad$ Mountain & 20 & $1.7^{*}$ & 13 & $2.2^{*}$ & 7 & $1.2^{*}$ \\
$\quad$ Central & 14 & 1.4 & 10 & $2.0^{*}$ & 4 & 0.8 \\
$\quad$ Eastern & 11 & $2.6^{*}$ & 5 & $2.3^{*}$ & 6 & $2.9^{*}$ \\
\hline
\end{tabular}

*Higher than the national rate $(1.4 / 100,000)$.

The incidence rate of CNS tumours per 100,000 children was 1.4. This rate was higher in males than females (1.7 versus 1.1). Geographically, the Eastern region had higher rates than the other regions, particularly for females. 


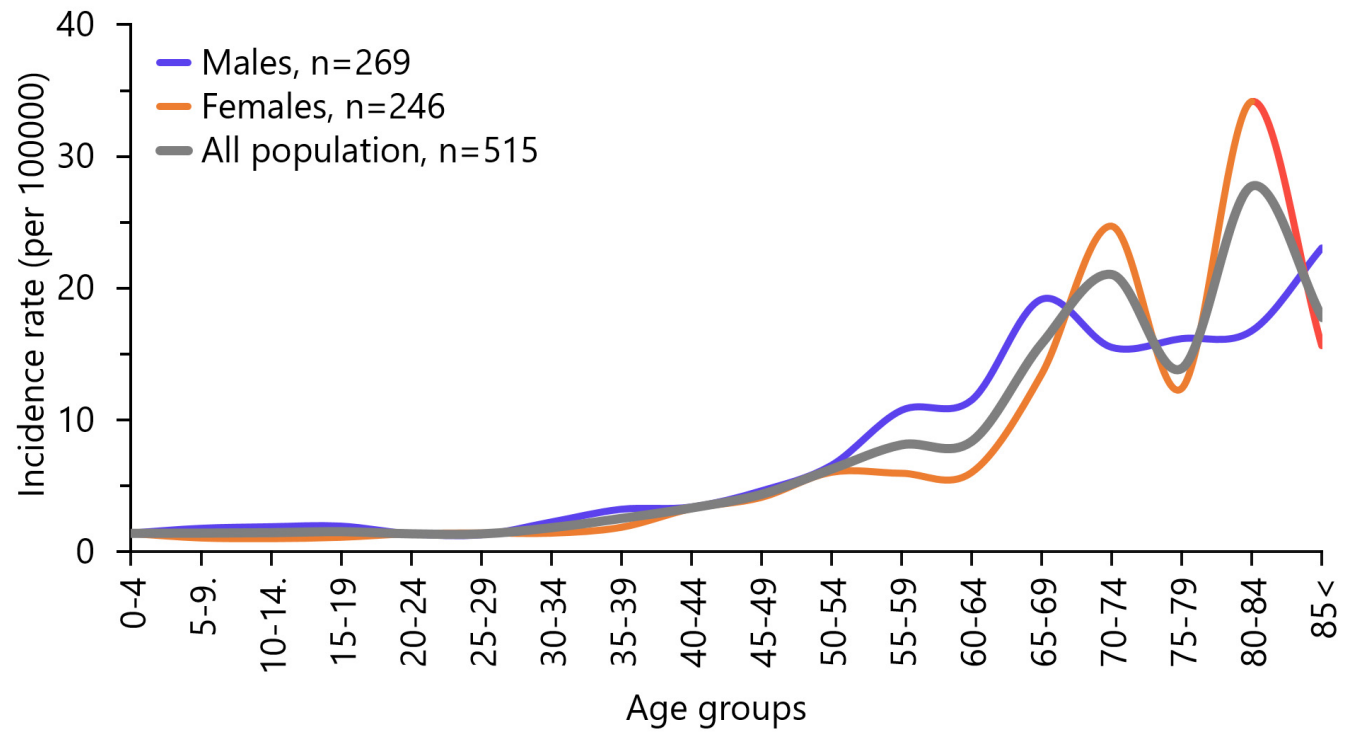

Fig. 3. Age-specific incidence of CNS tumours per 100,000 population by sex, 2015-2019 $(n=515)$.

Table 3. Distribution of CNS tumours by histology, 2015-2019 $(n=118)$.

\begin{tabular}{|c|c|c|c|c|}
\hline \multirow{2}{*}{ ICD-O-3 classification } & \multicolumn{2}{|c|}{ All population } & \multicolumn{2}{|c|}{ Children } \\
\hline & Cases & Percentage & Cases & Percentage \\
\hline Gliomas, malignant (938-948) & 68 & 57.6 & 18 & 72 \\
\hline Neuroepitheliomatous neoplasm (949-952) & 3 & 2.5 & 2 & 8 \\
\hline Meningioma, malignant (953) & 7 & 5.9 & - & 0.0 \\
\hline Nerve sheath tumours (954-957) & 6 & 5.1 & - & 0.0 \\
\hline Others (germ cell malignant, hemiangiopericytoma malignant, chondrosarcoma, malignant lymphoma) & 13 & 11.0 & - & 0.0 \\
\hline Neoplasm, unspecified & 21 & 17.8 & 5 & 20 \\
\hline Total & 118 & 100.0 & 25 & 100.0 \\
\hline
\end{tabular}

\subsubsection{Distribution of CNS tumours by histology}

Histologically confirmed diagnoses were $23 \%(n=118)$ of newly diagnosed CNS tumours in Mongolia. Among proven cases of CNS tumours glioma was the most prevalent histologic group (57.6\%) regardless of sex. Glioma accounted for $72 \%$ of all CNS tumours among children (Table 3 ).

\subsection{Age-standardized mortality rate}

From 2015 to 2019 a total of 381 deaths were registered including 196 males and 185 females. The national five-year mean annual ASMR was 3.0 per 100,000 population in Mongolia. This rate was higher in males than females (3.3 versus 2.6). The highest mean annual ASMR for males was in the Eastern region (5.6 per 100,000), followed by the Central region (4.6 per 100,000 males). In Ulaanbaatar, the mean annual ASMR was lower than the country average. The Eastern and Central regions had the highest rates, whereas the Western region had the lowest (Table 4).

\subsection{Survival rate}

283 cases were newly diagnosed during 2015-2017 and were included in the survival analysis. The three-year survival of all CNS tumors was $40.6 \%$ which represents 115 patients with CNS tumours who were alive three years after
Table 4. Five-year death cases and mean annual ASMRs by geographical region, 2015-2019 $(n=381)$.

\begin{tabular}{lcccccc}
\hline \multirow{2}{*}{ Characteristics } & \multicolumn{2}{c}{ All population } & \multicolumn{2}{c}{ Males } & \multicolumn{2}{c}{ Females } \\
\cline { 2 - 7 } & Cases & ASMR & Cases & ASMR & Cases & ASMR \\
\hline Mongolia & 381 & 3 & 196 & 3.3 & 185 & 2.6 \\
Urban region & & & & & & \\
$\quad$ Ulaanbaatar & 172 & 3 & 77 & 3 & 95 & $3^{*}$ \\
Rural region & & & & & & \\
$\quad$ Western & 34 & 2.2 & 18 & 2.2 & 16 & 2.1 \\
$\quad$ Mountain & 61 & 2.3 & 35 & 2.8 & 26 & 1.9 \\
$\quad$ Central & 80 & $3.8^{*}$ & 45 & $4.6^{*}$ & 35 & $3^{*}$ \\
$\quad$ Eastern & 34 & $3.8^{*}$ & 21 & $5.6^{*}$ & 13 & $2.6^{*}$ \\
\hline
\end{tabular}

*Higher than the national rate $(3 / 100,000)$.

diagnosis. 232 cases were excluded of which 186 were diagnosed in 2018-2019 and 46 were untraceable and it was not known whether they were alive or dead after three years. The type of treatment modality received was sorted into three groups. In the first group, 141 patients were treated with combined therapy of surgery, chemotherapy and radiotherapy and 81 (57\%) survived. In the second group, 13 patients were treated with either chemotherapy or radiotherapy and 
$6(46 \%)$ survived. In the third group, 129 patients were unresponsive or refused any treatment and 28 of them (22\%) survived. A log-rank test showed that the survival distributions for the three treatment modalities were significantly different $\left(\chi^{2}(1)=12.76, p<0.001\right)$. Kaplan-Meier survival curves showed the mean survival time for the first group was 12 months, for the second group 6 months and for the third group 5 months, respectively (Fig. 4).

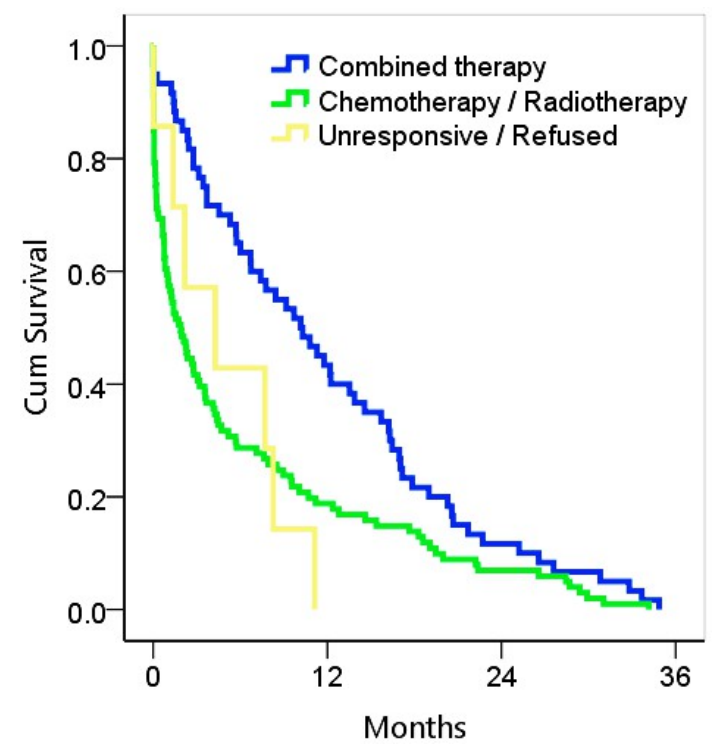

Fig. 4. Three-year survival rate by treatment modalities, 2015-2017 ( $n$ =283).

There was a total of 183 cases registered as alive during the period between 2015 and 2019 regardless of diagnosis dates. The five-year prevalence for all CNS was estimated to be 5.5 per 100,000 population.

\section{Discussion}

This is the first study on the epidemiology of CNS tumours in Mongolia based on nationwide registry data. The age-standardized incidence rate for CNS tumours in Mongolia was found to be 3.7 per 100,000 population during 20152019. The annual incidence rate in children aged from birth to 19 years was 1.4 per 100,000 . Although earlier studies reported that the ASR of total cancer increased from 171.3 to 238.6 per 100,000 in males and from 103.2 to 174 per 100.000 in females between 1973 and 1999 in Mongolia, the rate of CNS tumours was not listed [10-13]. Incidences in both sexes increased from the age of 40 years with advancing age. This rate was higher in males than females. In Mongolia the most common location of CNS tumours was the brain regardless of sex and age. In contrast, the meninges were the most common location in other large registries such as Korea and the United States of America (US) [17, 18]. However, those registries collect data on non-malignant tumours and the meningeal tumours were most likely non-malignant.
CNS incidence in Mongolia seems to be in a lower range when compared with other Asian nations or the Asian population in the US [5, 19-23]. This relatively low incidence of CNS tumours found in Mongolia may be explained by the absence of non-malignant CNS tumours data in the registry compared to other larger registries such as the US Surveillance, Epidemiology, and End Results Program (US-SEER) or the Scandinavian registries [20,24]. This difference may reflect the expansion of cancer incidence after the shift of the Korean Central Cancer Registry from the hospital-based to a population-based registry [25]. The shapes of age-specific curves of incidence rates were similar to those depicted from the other well-established cancer registries in Asia [26]. Although the cause of this significant geographical variation in the incidence of CNS tumours is unknown, several studies suggested that genetics, ethnicity, lifestyle, stress and environment may be responsible for the differences [5, 20, 23]. It suggests that the trend of increase in the incidence rate of CNS tumours is associated with the development of imaging techniques, however, it is not similar to that in developing countries like Mongolia [5, 20, 23].

The most commonly occurring CNS tumour among histopathology proven cases was glioma both in adults and children (57.6\% for all populations, $72 \%$ for children). This is concordant with the global pattern but unlike other Asian countries where meningioma is the most common CNS tumour [5, 23]. Histologically confirmed diagnoses were 23\% of newly diagnosed CNS tumours in Mongolia which is relatively low when compared to that of $58 \%$ in Korea, $73 \%$ in the US, and $86 \%$ in Japan. The main reason for the very low number of histologically confirmed diagnoses may be related to the uncoordinated report system for registration of the results of histological examinations in tertiary health care settings. Most patients with CNS tumours receive surgical treatment in the department of neurosurgery of TGHM but the pathology laboratory does not have to send the results to NCRM. The pathology laboratory at TGHM had four pathologists and four technicians. Since 2019, the laboratory has moved to NPC which currently has 14 pathologists, 23 technicians, and 2 assistant staff. Histology codes are only assigned by pathologists and this histopathology information is available only in the patient's medical record book. NPC only transmits a summary of the histologic examinations to HDCM and not to NCRM. It also does not provide information on each examination by a patient's unique registration number. Therefore, most CNS tumours were registered without histological verification at NCRM. There is no coordinated reporting system between NCRM and NPC regarding histologic examinations, so no available data source for the collection of histologic examinations for CNS tumours in Mongolia via NCRM. Generally, no mechanism currently exists for central review of diagnostic procedures including either histologic or radiographic confirmation for tumours, including CNS tumours, in the country. Therefore, information on current biomarkers for CNS tumours or genomic 
profiling is not available at NCRM. Moreover, private hospitals that provide spinal surgeries do not report whether there was a CNS tumour to NCRM. In addition to this major issue regarding the report system, non-malignant CNS tumours are not registered anywhere in Mongolia. Neurologists, neurosurgeons and psychiatrists often refer patients with headaches or seizures to radiological testing. However, symptoms like headaches are nonspecific and only a few patients with headaches have CNS tumours [18]. The nonavailability of advanced imaging and radiologists, neurologists, oncologists and neurosurgeons clearly affects diagnostic accuracy and therefore also registry and death certificate data.

381 deaths were registered as CNS tumours between 2015 and 2019 and the corresponding age-standardized mortality rate was 3.0 per 100,000 population. This value is lower than the rate in the US [23]. The five-year prevalence for all CNS turours is estimated to be 5.5 per 100,000 population. Survival outcomes depend on many factors including histologic types, diagnosis accuracy and availability of treatment modalities. The three-year survival rate for CNS tumours in Mongolia was found to be $36.6 \%$, which is not appropriate for comparison with previous studies of five-year survival rates. Nevertheless, it was close to the five-year survival rate in the United States (35.8\%) and even lower than found in Korea (44.1\%). The mortality to incidence ratio which can be seen as a crude indicator for survival was found to be 0.74 (381/515). This is close to the world average of 0.72 which was estimated by Globocan from 287,893 incident cases and 208,163 deaths in 2012 [5]. The relatively low survival outcomes indicate that the effects of treatment available in Mongolia are not sufficient. The main reason might be that there are no advanced treatment modalities such as gamma knife, cyberknife, proton-beam therapy or photon-beam therapy. Additionally, treatment for CNS tumours is expensive and usually takes a long time. In Mongolia, patients must pay outof-pocket $30 \%$ of the entire amount of treatment cost. Health insurance covered $70 \%$ of the cost for conventional therapies including neurosurgery, regular radiation therapy and palliative care until 2020 and now covers $100 \%$ of the cost. However, due to the low survival rate of those conventional therapies, with no advanced adjuvant and neoadjuvant therapies available in Mongolia, patients with CNS tumours who were capable of paying the high cost of treatment seek medical care abroad. In 2017, inoperable CNS tumours were included in the official list of untreatable diseases in Mongolia. Although health insurance covers treatment cost, people have to pay almost the entire amount of diagnostic cost after the first visit. For palliative care, health insurance partially covers inpatient care.

Taken together, the findings of this study demonstrate that there are the following issues in the care for CNS tumours in Mongolia: (1) no collection of data on nonmalignant CNS tumours which are characterized by lifethreatening clinical features; (2) no collection of data on precise locations for CNS tumours; (3) a very low rate of his- tological verifications; (4) no collection of data on outcomes of CNS tumour cases which were treated abroad; (5) no research reports on epidemiological, prognostic and therapeutic features of CNS tumours; (6) no availability of advanced treatment modalities for CNS tumours such as gamma knife or external-beam therapy; and (7) no availability of neoadjuvant therapies for CNS tumours. The issues identified in this study may exist not only for the care of CNS tumours but also for nationwide cancer care. Therefore, for national health policy and health care assessment to improve the diagnosis, prognosis and treatment of CNS tumours, expanding the cancer registry through collecting data on non-malignant tumours and the subtypes of CNS tumours, raising the rate of histological verification by clarifying diagnostic and reporting guidelines in line with international standards, conducting studies on health care service and cancer epidemiology regularly and introducing advanced treatment technologies for CNS tumours along with strengthening the human resource are recommended.

This study has several notable limitations: (1) no data on non-malignant CNS tumours; (2) a low rate of histologically confirmed diagnoses; (3) a short-term period for survival analysis that makes this data inappropriate for comparison with other studies; (4) limited information on precise anatomical sites of CNS tumours; and finally (5) a relatively high proportion of unspecified CNS tumour sites.

\section{Conclusions}

In summary, the data from the National Cancer Registry indicate that the incidence and survival rates of CNS tumours in Mongolia are relatively low. The most common location of CNS tumours was the brain. Glioma was the most common CNS tumour among histology confirmed cases. Despite its limitations, data from this study should provide information for national health policy and health care assessment. To improve the diagnosis, prognosis and treatment of CNS tumours, expanding the cancer registry through collecting data on non-malignant CNS tumours, raising the rate of histological verification by clarifying diagnostic and reporting guidelines in line with international standards, conducting studies on health care service and cancer epidemiology regularly and introducing advanced treatment technologies for CNS tumours along with strengthening the human resource are recommended.

\section{Abbreviations}

CNS, central nervous system; ICD, International Classification Disorders; ASR, age-standardized incidence rate; ASMR, age-standardized mortality rate; NCC, national cancer center; CT, computer tomography; NCRM, national cancer registry of Mongolia; NPC, national pathology center; TGHM, the third general hospital of Mongolia; HDCM, health development center of Mongolia; Globocan, Global Cancer Incidence, Mortality, and Prevalence; US-SEER, US Surveillance, Epidemiology, and End Results Program. 


\section{Author contributions}

BL conceived and designed the study; TS and UT performed the data collection; TS analyzed the data; UT contributed materials; TS, OJ, TO, and BL wrote the paper.

\section{Ethics approval and consent to participate}

Our study was conducted in accordance with the ethical standards outlined in the 1964 World Medical Association Declaration of Helsinki. The institutional review board and Ethics committee of the Mongolian National University of Medical Sciences approved the study protocol on May 3, 2020 (Ethics Nr. 20/06-12).

\section{Acknowledgment}

We thank the anonymous reviewers of our manuscript for their excellent criticism and constructive comments.

\section{Funding}

This study was supported by the IBRO/HRF-2014.

\section{Conflict of interest}

The authors declare no conflict of interest.

\section{References}

[1] The World Bank W. World Bank Country Classification. The World Bank. 2021. Available at: https: //datatopics.worldbank.org/world-development-indicator s/the-world-by-income-and-region.html (Accessed: 15 June 2020).

[2] National Statistical Office of Mongolia. 2019 Population and Housing By-Census of Mongolia. 2020. Available at: https://ww w.1212.mn/Stat.aspx?LIST_ID=976_L03\&type=tables (Accessed: 15 December 2020).

[3] Lim M, Myagmarchuluun S, Ban H, Hwang Y, Ochir C, Lodoisamba D, et al. Characteristics of Indoor PM2.5 Concentration in Gers Using Coal Stoves in Ulaanbaatar, Mongolia. International Journal of Environmental Research and Public Health. 2018; 15: 2524.

[4] Health Development Center of Mongolia. 2019 Health Indicators Mongolia. 2020. Available at: http://hdc.gov.mn/file-category/ 13/ (Accessed: 11 December 2020).

[5] GBD 2016 Brain and Other CNS Cancer Collaborators. Global, regional, and national burden of brain and other CNS cancer, 19902016: a systematic analysis for the Global Burden of Disease Study 2016. Lancet Neurol. 2019; 18: 376-393.

[6] Miranda-Filho A, Piñeros M, Soerjomataram I, Deltour I, Bray F. Cancers of the brain and CNS: global patterns and trends in incidence. Neuro-Oncology. 2017; 19: 270-280.

[7] Ferlay J, Colombet M, Soerjomataram I, Mathers C, Parkin DM, Piñeros $\mathrm{M}$, et al. Estimating the global cancer incidence and mortality in 2018: GLOBOCAN sources and methods. International Journal of Cancer. 2019; 144: 1941-1953.

[8] Shin H, Carlos MC, Varghese C. Cancer control in the Asia Pacific region: current status and concerns. Japanese Journal of Clinical Oncology. 2012; 42: 867-881.

[9] Allemani C, Weir HK, Carreira H, Harewood R, Spika D, Wang X, et al. Global surveillance of cancer survival 1995-2009: analysis of individual data for 25676887 patients from 279 population-based registries in 67 countries (CONCORD-2). Lancet. 2015; 385: 9771010.
[10] Sandagdorj T, Sanjaajamts E, Tudev U, Oyunchimeg D, Ochir C, Roder D. Cancer incidence and mortality in Mongolia - National Registry Data. Asian Pacific Journal of Cancer Prevention. 2010; 11: 1509-1514.

[11] Chimed T, Sandagdorj T, Znaor A, Laversanne M, Tseveen B, Genden $\mathrm{P}$, et al. Cancer incidence and cancer control in Mongolia: Results from the National Cancer Registry 2008-12. International Journal of Cancer. 2017; 140: 302-309.

[12] Znaor A, Chimed T, Laversanne M, Tudev U, Sanjaajamts E, Sandagdorj $\mathrm{T}$, et al. The public health challenge of liver cancer in Mongolia. Lancet Gastroenterology \& Hepatology. 2019; 3: 660662.

[13] International Agency for Research on Cancer. GLOBOCAN Population fact sheets - Mongolia. 2020. Available at: https://gco.iarc.fr/today/data/factsheets/populations/496-mon golia-fact-sheets.pdf (Accessed: 15 May 2020).

[14] World Health Organization. ICD-10: international statistical classification of diseases and related health problems: tenth revision, 2nd ed. 2004. Available at: https://icd.who.int/browse10/2019/ en (Accessed: 15 June 2020).

[15] Fritz A, Percy C, Jack A, Shanmugaratnam K, Sobin L, Parkin DM, et al. International classification of diseases for oncology. 3 rd edn. World Health Organization: Malta. 2000.

[16] International Agency for Research on Cancer. CI5 Vol. XI, Chapter 7: Age standardization. 2020. Available at: https://ci5.iarc.fr/ ci5-xi/pages/chapter7.aspx (Accessed: 15 May 2020).

[17] Kang H, Song SW, Ha J, Won Y, Park C, Yoo H, et al. A Nationwide, Population-Based Epidemiology Study of Primary Central Nervous System Tumors in Korea, 2007-2016: a Comparison with United States Data. Cancer Research and Treatment. 2021; 53: 355-366.

[18] GBD 2015 Neurological Disorders Collaborator Group. Global, regional, and national burden of neurological disorders during 1990-2015: a systematic analysis for the Global Burden of Disease Study 2015. Lancet Neurology. 2017; 16: 877-897.

[19] Zheng R, Zeng H, Zhang S, Chen W. Estimates of cancer incidence and mortality in China, 2013. Chinese Journal of Cancer. 2017; 36: 66.

[20] Natukka T, Raitanen J, Haapasalo H, Auvinen A. Incidence trends of adult malignant brain tumors in Finland, 1990-2016. Acta Oncologica. 2019; 58: 990-996.

[21] Beygi S, Saadat S, Jazayeri SB, Rahimi-Movaghar V. Epidemiology of pediatric primary malignant central nervous system tumors in Iran: a 10 year report of National Cancer Registry. Cancer Epidemiology. 2013; 37: 396-401.

[22] Lee C, Jung K, Yoo H, Park S, Lee SH. Epidemiology of primary brain and central nervous system tumors in Korea. Journal of Korean Neurosurgical Society. 2010; 48: 145-152.

[23] Ostrom QT, Cioffi G, Gittleman H, Patil N, Waite K, Kruchko C, et al. CBTRUS Statistical Report: Primary Brain and other Central Nervous System Tumors Diagnosed in the United States in 2012 2016. Neuro-Oncology. 2019; 21: v1-v100.

[24] Surveillance, Epidemiology, and End Results (SEER) Program (www.seer.cancer.gov) SEER*Stat Database: Incidence - SEER Research Data, 9 Registries, Nov 2020 Sub (1975-2018) Linked To County Attributes - Time Dependent (1990-2018) Income/Rurality, 1969-2019 Counties, National Cancer Institute, DCCPS, Surveillance Research Program, released April 2021. Available at: https://seer.cancer.gov/data/citation.html (Accessed: 15 June 2021).

[25] Shin H, Won Y, Jung K, Kong H, Yim S, Lee J, et al. Nationwide cancer incidence in Korea, 1999 2001; first result using the national cancer incidence database. Cancer Research and Treatment. 2005; 37: 325-331.

[26] International Agency for Research on Cancer. Cancer Incidence in Five Continents Volume XI. 2020. Available at: https://ci5.iarc .fr/CI5-XI/ (Accessed: 15 May 2020). 